\title{
Measuring community/environmental interventions: the Child Pedestrian Injury Prevention Project
}

\author{
Mark Stevenson, Helena Iredell, Peter Howat, Donna Cross, Margaret Hall
}

\begin{abstract}
Objectives-To assess the effectiveness of community/environmental interventions undertaken as part of the Child Pedestrian Injury Prevention Project (CPIPP). Setting-Three communities (local government areas) in the Perth metropolitan area, Western Australia.

Methods-A quasiexperimental community intervention trial was undertaken over three years (1995-97). Three communities were assigned to either: a community/environmental road safety intervention and a school based road/ pedestrian safety education program (intervention group 1); a school based road/pedestrian safety education program only (intervention group 2); or to no road safety intervention (comparison group). Quantification of the various road safety community/environmental activities undertaken in each community during the trial was measured, and a cumulative community activity index developed. Estimates of the volume and speed of vehicular traffic were monitored over a two year period.
\end{abstract}

Results-Greater road safety activity was observed in intervention group 1 compared with the other groups. A significant reduction in the volume of traffic on local access roads was also observed over the period of the trial in intervention group 1 , but not in the remaining groups.

Department of Epidemiology and Biostatistics, School of Public Health, Curtin University of Technology, Perth, Australia

M Stevenson

Centre for Health Promotion Research, School of Public

Health, Curtin

University of

Technology, Perth,

Australia

$\mathrm{H}$ Iredell

P Howat

D Cross

M Hall

Correspondence to: Dr M Stevenson, NCIPC, Centers for Disease Control and Prevention, Mailstop K63, 4770 Buford Highway NE, Atlanta, GA 30341-3724, USA the various community/environmental interventions initiated in collaboration with CPIPP in intervention group 1 contributed, in part, to the observed reduction in the volume of traffic. A combination of community/environmental interventions and education are likely to reduce the rate of childhood pedestrian injury.

(Injury Prevention 1999;5:26-30)

Keywords: Child Pedestrian Injury Prevention Project; pedestrians; interventions; community/environment

In Western Australia, pedestrian injuries are the leading cause of injury death in children aged 5-9 years ${ }^{1}$ and these findings are found in other parts of Australia and most western countries. ${ }^{2-6}$ The average length of hospital stay after a pedestrian injury for children in this age group is four times longer than that for a burns victim. ${ }^{7}$ There is evidence that success in preventing child pedestrian injuries in some
European countries can be attributed to environmental changes implemented as part of planned programs of injury prevention. ${ }^{8}$ Environmental approaches are intended to impose barriers between motor vehicles and pedestrians, or to modify the speed and volume of traffic in areas used by pedestrians. ${ }^{9}{ }^{10}$ Findings from previous research support, as priority initiatives, modifications to the physical environment in terms of the calming of vehicular traffic as well as changes in the streetscape to ensure permanent structures minimise obscuration of children. ${ }^{11}$ Recent research indicates that the first of these approaches, the calming of vehicular traffic, is a cost effective intervention. ${ }^{12}$

To assess the effectiveness of community/ environmental interventions along with school based road safety education, a three year community intervention trial-the Child Pedestrian Injury Prevention Project (CPIPP) - was initiated. The aim of CPIPP was to increase 6-9 year old children's: road safety knowledge; to improve their road crossing behaviour; and to modify identified risks in the road environment. This is the first, comprehensive community intervention trial that has implemented an extensive school based education program as well as actively engaged the community in environmental strategies to reduce a child's risk of injury in the road environment.

This paper describes the processes undertaken to determine the intensity and perceived benefits of various community/environmental interventions, as well as the extent to which the community/environmental interventions were successful.

\section{Methods}

Three local government areas in the Perth metropolitan area (population 1.3 million), Western Australia were selected based on similar childhood pedestrian injury rates, their sociodemographic characteristics, and that they were at a sufficient distance apart (geographically) so as to minimize contamination of the interventions. The local government areas were then assigned to one of three conditions. The first local government area (intervention group 1) received both a school based community/environmental interventions. The school based road safety education program was implemented during three of the school terms in each of the three years of the trial (1995-97). Details of the program are documented elsewhere. ${ }^{13}$ road safety education program and 
The community/environmental interventions, which were also implemented over the three year period, comprised lobbying for changes to speed limits; development of traffic calming features; community education campaigns; and the establishment of Safe-Routesto-School programs. The Safe-Routes-toSchool program was established with the support of each school and involved mapping safe routes, in terms of least hazardous traffic, for children to follow when walking to school. A community advisory committee was established to facilitate the various activities. The committee comprised the city traffic engineer, a city councillor, two advocates for road safety, a police officer, two community residents, a representative from the government department, Main Roads Western Australia, and two CPIPP representatives. Further details on the community/environmental interventions are reported elsewhere. ${ }^{14}$ The second local government area (intervention group 2), received the same school based road safety education program over the three years of CPIPP but no community/environmental interventions. The third local government area (comparison group) did not receive either the school based road safety education program or the community/environmental interventions. Instead, they received a school based nutrition education program over the same period.

To quantify the magnitude of the CPIPP community/environmental interventions as well as local government road safety initiatives across all three communities over the three years, it was necessary to develop an index of community activity. The index was developed in three stages. The first stage involved recording details of various activities that either reduced the risk of child pedestrian injury in the road environment or heightened the community's awareness of road safety over the three year period of the trial. This was undertaken by reviewing the local government records in the three communities. Researchers spent time, at the completion of the trial, in the local government offices of each community, transcribing, onto standardised forms (instruments used in this study are available from the authors), various activities undertaken in each community over the period of the intervention (1995-97). Information on activities such as the installation of traffic calming measures on local roads; the construction of footpaths for use by pedestrians; installation of pedestrian related road signs; and the installation of pedestrian refuges, were recorded from the government records. Key contacts in each community, namely the road safety coordinators, were also approached to provide details on activities that would not have been recorded in the local government records such as road safety related displays or promotions in shopping centres. The road safety coordinators also verified the accuracy of the activities that were transcribed from the office records.

For each activity, information on its duration was collected on the basis of calendar months. Consequently, a community/environmental activity could run for as little as one month (for example, road safety displays in shopping centres) to as much as 36 months. An example of an activity that could run for 36 months would be the installation of a roundabout that was installed in the first month of year 1 of the trial.

The second stage involved a survey to canvass the public's opinion of various community/environmental interventions. That is, the public's perception of whether the various activities would lead to changes in road safety behaviour. Behaviours such as reducing vehicular speed and volume which are likely to reduce the risk of childhood pedestrian injury. To obtain the public's opinion, the Delphi technique was used. ${ }^{15}$ This provided an economically viable way of achieving a broad analytical perspective of the public's perception of the various community/environmental road safety activities.

A questionnaire was developed and posted to a cross section of the public $(n=25)$; this comprised university academics, engineers, teachers, parents of young children, administrators from the public service, students, health professionals, and psychologists. The questionnaire described $25 \mathrm{road} / \mathrm{road}$ safety related activities in lay terms with definitions provided in an appendix. Attached to each activity was a visual analogue scale that ranged from "no change in behaviour" to "maximum change in behaviour". Each respondent was asked to place a "cross" along the analogue scale that best indicated their perception of the behaviour change likely to occur due to the activity. The value was recorded by measuring, in $\mathrm{cm}$, the distance between the "cross" and the mark indicating "no change in behaviour". A rating ranging from $1-10$ (measured in $\mathrm{cm}$ ) was assigned to each activity indicating the respondent's perception of the likely behaviour change.

In the final stage, a score for each road safety activity initiated in intervention groups 1 and 2 and the comparison group, was calculated by combining the duration of the activity over the period of the trial, with the findings from the Delphi technique for the same activity. For example, the introduction in May 1996 of traffic calming facilities in a local street in intervention group 1, lead to a community/ environment score of 123.5 (out of a possible 360). This score is the multiplication of the duration of the activity (19 months) and the public's perception of the activity (score of 6.5 out of a possible 10).

The community/environment score for each activity was summated across all activities to give a cumulative dose of community activity for intervention groups 1 and 2 and the comparison group. Since the total population and land area differed significantly between the three communities, each community/ environmental dose score was adjusted for the total kilometres of road and the total number of residents in the community.

As alluded to earlier, only intensive community/environmental interventions were implemented in intervention group 1. To detect whether the various community/ environmental interventions met the stated 
Table 1 Public's perception of road safety activities

\begin{tabular}{ll}
\hline Highest and lowest ranked activities & $\begin{array}{l}\text { Mean (SD) score } \\
\text { (maximum score }=10)\end{array}$ \\
\hline Provision of school crossings & $8.8(1.12)$ \\
Lowering of speed limits in school zones & $8.4(1.36)$ \\
Establishment of "Safe Routes to School' program & $8.1(1.88)$ \\
Providing drop-off/pick-up zones for parents adjacent to school & $7.9(1.85)$ \\
Road safety competitions in the local community & $5.1(2.46)$ \\
Conducting road safety displays in shopping centres & $4.3(2.54)$ \\
Widening of the road & $4.0(2.66)$ \\
Installation of traffic lights without a pedestrian walk sign & $3.3(2.60)$ \\
\hline
\end{tabular}

objective, namely, to modify risks in the road environment, it was necessary to assess the volume and speed of vehicular traffic over the three year period. A stratified random sample of 135 roads in intervention groups $1(n=45), 2$ $(n=45)$, and the comparison group $(n=45)$ were selected. The roads were stratified into two levels (local access and local distributor roads) and the average five day speeds and volumes were recorded on the same roads at two points: baseline 1995 and at post-test 1996 over the period of the trial. The volumes and speed of traffic were recorded over similar four month periods and the data were collected using a pneumatic vehicle classifier (MetroCount 3500 Traffic Analysis software, Microcom).

Descriptive statistics described the distribution of the cumulative dose of community activity between the three communities. Also, means and SDs were presented for the public's perception of the various road safety activities. The dependent variables, namely, vehicular speed and volume, were assessed to determine whether they changed from baseline to follow up between the three communities. Using generalized linear modelling, we conducted two repeated measures analysis of variances using time (baseline 1995, and follow up 1996), group (whether intervention group 1 or 2 or the comparison group), and time and group interaction as fixed effects. All computations were undertaken using SPSS ${ }^{16}$ and SAS ${ }^{17}$ software.

\section{Results}

The response rate from the postal questionnaire that elicited the public's perception of the various road safety activities was $80 \%$. The findings from the survey highlighted that activities conducted around schools were perceived to be of greatest value. Activities such as provision of school crossings, lowering of speed limits in school zones, and providing

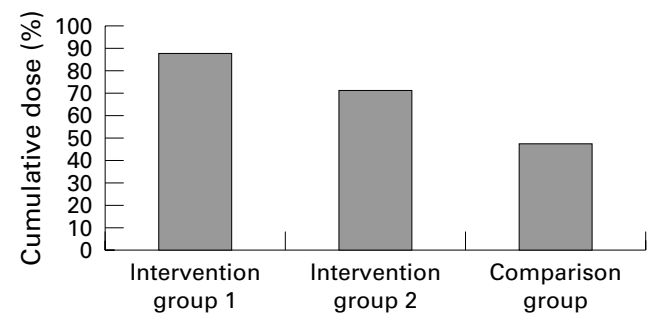

Figure 1 Cumulative dose of community activity (1995-97).

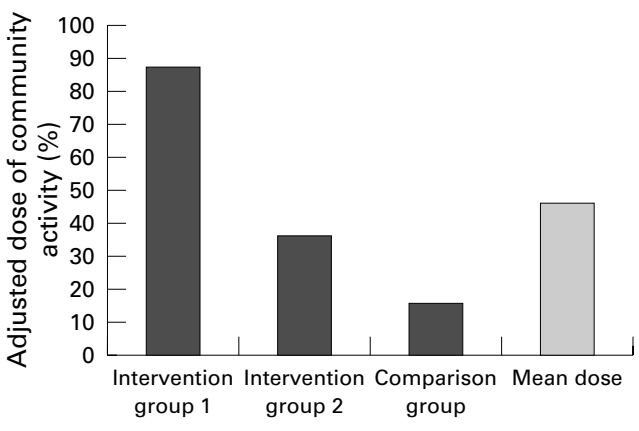

Figure 2 Cumulative dose of community activity (1995-97), adjusted for total $\mathrm{km}$ of road.

drop-off/pick-up zones for parents adjacent to school were ranked in the top four activities. As shown in table 1 , the activities that were perceived by the public as having least value were road safety displays in shopping centres and minor structural changes to the road.

Table 2 lists the 24 different community activities that were identified from the reviews of the local government records. As expected, intervention group 1 received the greatest cumulative dose of community activity over the duration of CPIPP (see fig 1). An assessment of the individual activities implemented in each community identified two activities, namely the Safe-Routes-to-School program and the installation of pedestrian refuges that were frequently implemented in intervention group 1 . The Safe-Routes-to-School program was 14 times and the installation of pedestrian refuges three times as likely to be implemented in intervention group 1 compared with intervention group 2 and the comparison group.

A substantial difference was detected in intervention group 1 compared with intervention group 2 and the comparison group when the dose of the community activity was adjusted for the total kilometres of road in each of the three communities (see fig 2). In fact, intervention group 1 had almost twice the dose

Table 2 Description of the environmental interventions

\author{
Road safety activities \\ Placement of pedestrian related signs \\ Lowering of speed limits in school zones \\ Construction of footpaths \\ Construction of dual use paths \\ Installation of traffic calming measures on local roads \\ Construction of roundabouts at local intersections \\ Installation of concrete pedestrian refuges \\ Installation of painted median islands \\ Placement of pedestrian handrails at crossing points \\ Installation of traffic lights with a pedestrian WALK signal \\ Provision of school crossing with attendant \\ Establishment of Safe-Routes-to-School program
}

Reduction of speed limit on local streets Closure of a through-road into a cul-de-sac

Provision of parent parking bays at schools

Narrowing of road to reduce speed

Conducting road safety displays in shopping centers

Establishment of a local road safety committee

Publishing articles on road safety in community newspaper

Disseminating written material on road safety to schools and communities

Holding a road safety day

Carrying out road safety competitions in the community

Installation of zebra crossings

Installation of an overpass 
Table 3 Per cent change in the volume of traffic

\begin{tabular}{|c|c|c|c|c|c|}
\hline Road type & Intervention group 1 & Intervention group 2 & Comparison group & $F$ value $(d f)$ & $p$ Value \\
\hline Local access roads (lower traffic volume) & $9 \% \downarrow(n=15)$ & $6 \% \uparrow(n=27)$ & Stable $(n=10)$ & $3.58(49)$ & 0.03 \\
\hline Local distributor roads (higher traffic volume) & Stable $(n=16)$ & $9 \% \downarrow(n=16)$ & $4 \% \uparrow(\mathrm{n}=6)$ & $0.98(35)$ & 0.39 \\
\hline
\end{tabular}

Table 4 Per cent change in the speed of traffic

\begin{tabular}{|c|c|c|c|c|c|}
\hline Road type & Intervention group 1 & Intervention group 2 & Comparison group & $F$ value $(d f)$ & $p$ Value \\
\hline Local access roads (lower traffic volume) & Stable $(n=14)$ & Stable $(n=27)$ & $6 \% \downarrow(n=10)$ & $6.73(47)$ & 0.00 \\
\hline Local distributor roads (higher traffic volumes) & $4 \% \uparrow(n=14)$ & $4 \% \uparrow(\mathrm{n}=16)$ & $5 \% \downarrow(\mathrm{n}=6)$ & $2.00(33)$ & 0.15 \\
\hline
\end{tabular}

or 1.8 times greater community activity than might be expected as an average level of activity in a community over three years (calculated as the average activity of the three communities).

A significant reduction in the volume of traffic on local access roads was detected in intervention group 1 compared with intervention group 2 and the comparison group (see table 3). Over a two year period (1995-96), a reduction in the volume of traffic of approximately $9 \%$ was observed in intervention group 1 . However, no differences were detected between the three groups on local distributor roads (roads with greater volumes of traffic).

Table 4 shows that the speed of traffic on local access roads remained the same over time in intervention groups 1 and 2, but reduced, significantly, in the comparison group. Like the volume of traffic, there was no significant reduction in the speed of traffic over time on local distributor roads across the three groups.

\section{Discussion}

The level of community/environmental activity evident in intervention group 1 was 2.3 times greater and 5 times greater when compared with intervention group 2 and the comparison group, respectively. As stated earlier, intervention group 1 was the only group that received community and environmental interventions over the three years of the trial. Therefore, can the greater level of community/environmental activity in intervention group 1 be attributed to the CPIPP intervention?

It is likely that part of the increased community activity in intervention group 1 could be an artefact of differential reporting. We attempted to minimise this bias when reviewing the local government records in each community by using the same independent observer and transcribing the data, in a systematic way, onto standardised forms. Notwithstanding this, the record keeping of local government activities was beyond our control, and therefore, each local government may have either underreported or over-reported their activities. Anecdotal evidence suggests, however, that intervention group 1 was less likely to over-report their road safety activities compared with intervention group 2 and the comparison group.

A further explanation for the raised cumulative dose of community activity in intervention group 1 could be due, in part, to the demographic and geographic differences between the three communities. This explanation is feasible given that intervention group 1 has a larger resident population than intervention group 2 for example, whereas intervention group 2, has a geographic area that is eight times larger with twice the total kilometres of road than intervention group 1 . We were aware of these differences, and, after adjusting the cumulative dose of community activities for either the total number of residents (not reported here), or the total kilometres of road, the increased level of community/ environmental activity remained higher in intervention group 1.

A final point in relation to the cumulative dose of community activity relates to the development of the indice. The weighting which comprised part of the dose measure relied on the findings from the Delphi technique which comprised a sample of 25 . Although this sample represented a wide cross section of the community, the insufficient numbers mean the findings from the survey may lack generalizability. Consequently, the estimates of cumulative dose are particularly crude.

Notwithstanding the above explanations, it is also important to note that there were existing road safety activities in intervention group 1 other than those related to CPIPP which may have contributed to the cumulative dose of community activity. To determine to what extent these may have affected each of the groups differently, we monitored newspaper articles, legislative changes, and other external factors over the period of the trial. These observations highlighted that the majority of activities not related to CPIPP, such as lowering speed limits around schools from 60 $\mathrm{kph}$ to $40 \mathrm{kph}$, occurred due to legislative changes at state rather than at local government level. Therefore, it is unlikely that external factors affected the three communities differently.

Assuming that differential reporting and/or other unobserved external factors account for approximately half of the raised cumulative dose of community activity in intervention group 1, it is reasonable therefore, that the remaining difference in activity may be directly attributed to the influence of CPIPP. That is, CPIPP may have accounted for an increase in road safety activity of approximately $50 \%$.

One of the objectives of CPIPP was to reduce the volume of traffic. Over a two year period, we observed a $9 \%$ reduction in the volume of traffic on local streets in intervention group 1. The most likely explanation for this reduction in intervention group 1 and not in the other communities, is the influence of 
CPIPP. An important finding from earlier research suggests that by reducing the volumes of traffic on local streets by $15 \%$, the incidence of child pedestrian injury could be reduced by up to $30 \%$. $^{18}$ Applying the same assumptions, a $9 \%$ reduction in the volume of traffic observed in intervention group 1 in this study could lead to a reduction in the incidence of childhood pedestrian injury by up to $18 \%$.

A number of limitations are apparent in relation to the data on the volume and speed of vehicular traffic. The first is that relatively few roads were surveyed. As would be expected, it was both time consuming and costly to observe a random sample of 135 roads over a period of five days for each of the three years. Consequently, we were only able to observe 90 roads. Due to the imposed constraints, we were unable to observe district distributor roads. However, since the child's exposure to the risk of injury is greatest on the roads observed, ${ }^{11}$ the absence of observations on district distributor roads is unlikely to have affected the interpretation of our findings. The second limitation of the reported data on the volume and speed of vehicular traffic was the lack of follow up data in 1997. The reason for not observing the roads in the final year was, again, due to financial constraints. In the absence of these data, we are unable to determine whether the significant change in the volume of traffic observed in intervention group 1 from 1995 to 1996 was sustained through to 1997 . This limitation needs to be considered when interpreting the results.

For future preventive strategies, it would have been useful to know what aspects of the environmental/community activities were most effective. However, this question was beyond the stated objectives of the study. Consequently, cumulative doses rather than activity specific doses were reported. To have obtained details on specific activities would have required a sample substantially larger than the three communities selected for CPIPP, and considerably more resources.

A further limitation of the data surrounds the issue of reliability and the validity of the various road safety activities that were reported. Again, due to financial and time constraints, we did not undertake these important procedures. Despite this, we have anecdotal evidence from the research staff that traffic calming initiatives reported in the local government records were in fact initiated.

\section{Implications for prevention}

The findings from this research indicate that the various community/environmental interventions implemented as part of CPIPP may contribute to a reduction in the volume of traffic on local streets. Moreover, this reduction in the volume of traffic did not result in increased speeds on the local streets. Research has shown that reducing the volume of traffic in local streets leads to a reduction in the rate of pedestrian injury. ${ }^{8}$ The rates of childhood pedestrian injury were not measured in this study due to the small numbers in each community. However, if the environmental and educational changes (reported elsewhere ${ }^{13}$ ) observed as a consequence of the CPIPP are sustained, it is likely that a reduction in the rate of child pedestrian injury in the community will occur.

The evidence to date suggests that the effects from the intervention will be sustained. At the time of writing, the school based road safety education materials, developed for CPIPP, have been incorporated in the health education curriculum throughout the state of Western Australia. Further, the Community Advisory Committee established to oversee the CPIPP environmental/community activities in intervention group 1 continues to function and implement various CPIPP activities.

We would like to thank Mr Jeff Brooks and the numerous staff and students from the School of Public Health, Curtin University and the school students, teachers, parents, and local government staff who generously assisted with this research. The Child Pedestrian Injury Prevention Project received financial support from the following agencies: the Western Australian Health Promotion Foundation, the Traffic Board of Western Australia, and Main Roads of Western Australia.

1 Health Department of Western Australia. Injury in Western Australia, 1983-1989. Perth, Western Australia: Health Department of Western Australia, 1991

2 Better Health Commission. Looking forward to better health Vol 2, part 3. Injury Taskforce report. Canberra: Australian Government Printing Service, 1986

3 Foot H, Chapman A, Wade F. Pedestrian accidents: general issues and approaches. In: Chapman A, Wade F, Foot H, eds. Pedestrian accidents. Chichester: John Wiley, 1982.

4 Rivara F. Child pedestrian injuries in the United States. $\mathrm{Am}$ f Dis Child 1990;144:692-6.

5 Roberts I, Norton R, Hassall I. Child pedestrian injury. $N Z$ Med F 1992;105:51-2.

6 Schelp L, Ekman R. Road traffic accidents in a Swedish Schelp L, Ekman R. Road traffic accidents
municipality. Public Health 1990;104:55-64.

7 Roberts I, Streat S, Judson J, et al. Critical injuries in paediatric pedestrians. NZ Med F 1991;104:147-8.

8 Bjaras G, Danielson K, Schelp L, et al. Safety rounds in public environment: experience of a new tool for prevention of accidental injuries. Accid Anal Prev 1990;22: 223-8.

9 National Committee for Injury Prevention and Control. (American Journal of Preventive Medicine.) Injury prevention; meeting the challenge. New York: Oxford University Press, 1989.

10 Alexander K, Cave T, Lyttle J. Pedestrian accident project. Report No 6. Melbourne: Road Traffic Authority of Victoria, 1990.

11 Stevenson M, Jamrozik K, Spittle J. A case control study of traffic risk factors and child pedestrian injuries. Int $\mathcal{F}$ Epidemiol 1995;24:1-8

12 Roberts I, Ashton T, Dunn R, et al. Preventing child pedestrian injury: pedestrian education or traffic calming. Aust $\mathcal{F}$ Public Health 1994;18:209-12.

13 Cross D, Stevenson M, Burns S, et al. The child pedestrian injury prevention project: evaluation of a school-based road safety education program. Prev Med (in press)

14 Gibbs S. The establishment and evaluation of the Gosnells Roadwise Committee. (Bachelor of Science honours dissertation.) Perth, Australia: Curtin University of Technology, 1996.

15 Duffield C. The Delphi technique. F Adv Nurs 1988;6:41-5. 6 Statistical Package for the Social Sciences. SPSS-X computer program. Chicago, IL: SPSS Inc, 1988.

17 Statistical Analysis System. SAS computer program. Cary, NC: SAS Institute Inc, 1990.

18 Stevenson M. Childhood pedestrian injuries: what can changes to the road environment achieve? Aust N Z F Public Health 1997;21:33-7. 